\title{
Equilibrium model for two low-pressure electronegative plasmas connected by a double layer
}

\author{
P. Chabert, ${ }^{\text {a) }}$ N. Plihon, C. S. Corr, and J.-L. Raimbault \\ Laboratoire de Physique et Technologie des Plasmas, Ecole Polytechnique, 91128 Palaiseau, France
}

\author{
A. J. Lichtenberg \\ Department of Electrical Engineering, University of California, Berkeley, California 94720
}

(Received 17 July 2006; accepted 7 August 2006; published online 21 September 2006)

\begin{abstract}
Plihon et al. [J. Appl. Phys. 98, 023306 (2005)] have recently shown that double layers usually form during the expansion of a low pressure electronegative plasma. These double layers act as permeable internal boundaries between the source (upstream) plasma and the downstream expanding plasma; positive ions flow from upstream to downstream whereas negative ions flow in the opposite direction. So far, the detailed physical mechanisms leading to their formation have not been identified. In this paper, we develop a model for the two plasma equilibria, upstream and downstream, assuming that the double layer exists and couples the two plasmas. At very low pressure, typically $0.5 \mathrm{mTorr}$, the coupling is strong and acts both ways. The negative ions created downstream contributes to the upstream equilibrium as well as the upstream positive ions contribute to the downstream equilibrium. As the pressure increases, the situation becomes asymmetric. The source plasma is not affected by the negative ions flowing from downstream, whereas the positive ions coming from the source control the downstream plasma equilibrium, where local ionization is negligible. () 2006 American Institute of Physics. [DOI: 10.1063/1.2345353]
\end{abstract}

\section{INTRODUCTION AND BACKGROUND}

Low-pressure electronegative discharges, using attaching gases, have drawn considerable attention in the last decade, not only because of their industrial potential (they are routinely used for the etching of semiconductors in the microelectronic and photonic industries ${ }^{1}$ ), but because their fundamental properties are significantly different from the more traditional electropositive discharge plasmas. Some of the most important and interesting problems are related to the existence of various kinds of instabilities and double layers, which have recently been observed and modeled in inductive and helicon discharges with electronegative gases. In such systems, the instabilities have been divided into two families: (i) source instabilities, which are related to the energy coupling modes and occur in the neighborhood of mode transitions (capacitive-to-inductive or inductive-to-helicon), ${ }^{2-6}$ and (ii) transport or downstream instabilites, occurring in the expanding region of the plasma. ${ }^{7-11}$ Double layers were also theoretically predicted and/or experimentally observed. They appeared to be either attached to the main sheath in onedimensional equilibrium calculations, as in Refs. 12-15, or to be internal sheaths sitting in the middle of the plasma, for instance, at the interface between the source and the diffusion region, as observed by Plihon et al. ${ }^{16}$ In some conditions, it was found that downstream instabilities were nonstationary double layers, periodically formed at one particular location and propagating in the expanding region. ${ }^{7}$

In this paper, we develop a model to describe the situation observed by Plihon et al., ${ }^{16}$ that is a stationary double layer connecting the source plasma to the downstream ex-

\footnotetext{
${ }^{a)}$ Electronic mail: chabert@1ptp.polytechnique.fr
}

panding plasma. Before describing the model (in Sec. II) and comparing it to experiments (in Sec. III), let us first summarize the experimental findings. Experiments were carried out in a low-pressure inductive discharge having a source region (a $15 \mathrm{~cm}$ diameter, $30 \mathrm{~cm}$ long, pyrex tube), connected to a cylindrical expanding chamber of similar length but two times larger in diameter. Various $\mathrm{Ar} / \mathrm{SF}_{6}$ mixtures, from pure argon to pure $\mathrm{SF}_{6}$, were investigated in order to probe the effect of changing the gas electronegativity. The pressure was typically varied from 0.5 to $10 \mathrm{mTorr}$ and the rf power range was typically $0-1000 \mathrm{~W}$. The important conclusions were the following.

- In pure argon, the discharge is stationary (no instabilities) and the plasma smoothly expands downstream (no double layers).

- As the $\mathrm{SF}_{6}$ percentage in argon (i.e., the electronegativity) is increased (for a typical pressure of $1 \mathrm{mTorr}$ ), the following sequence is observed: (i) from $0 \%$ to $8 \%$, no instabilities and no double layers, (ii) from $8 \%$ to $13 \%$, a stationary double layer forms at the interface between the source and the expanding chamber, which separates a low-electronegativity, high density, high electron temperature plasma upstream, from a highelectronegativity, low density, low electron temperature plasma downstream, (iii) above $13 \%$, the double layers become unstable, periodically forming at the interface and propagating downstream at low speed. The period is such that a second double layer forms before the first has reached the bottom of the chamber; i.e., for a given time during the period, there are two double layers in the expanding plasma.

- At 1 mTorr, the upstream electron temperature is about 


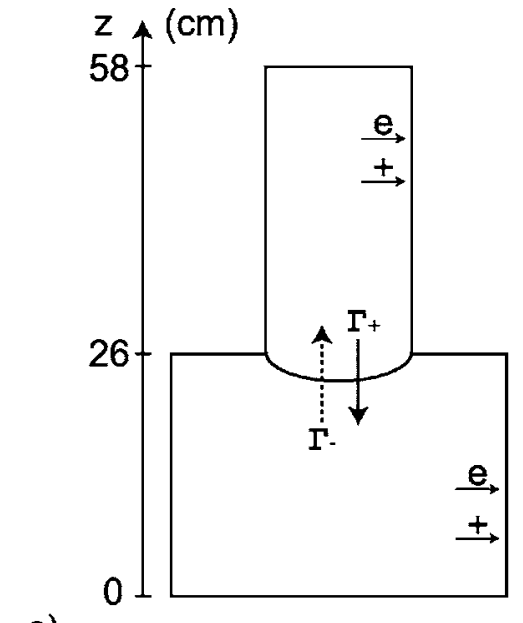

a)

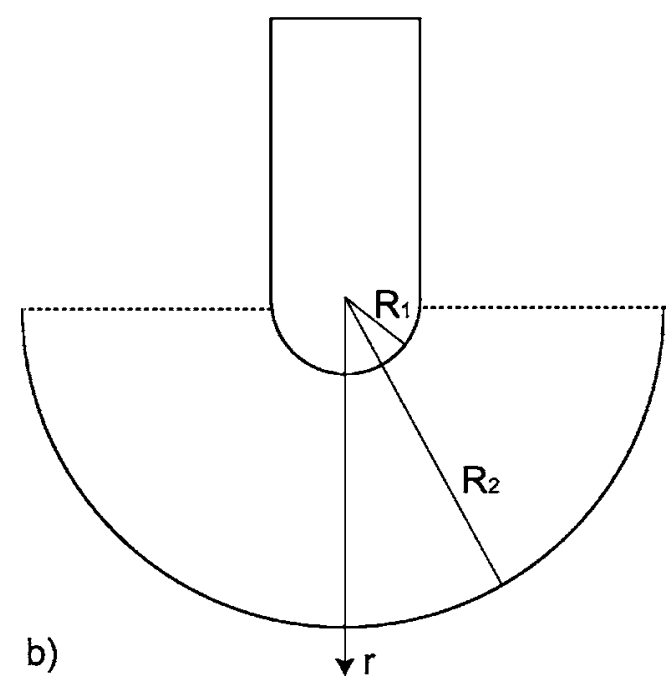

FIG. 1. Schematic of the experiments (a) and geometry used in the model (b)

$4.5 \mathrm{eV}$ and the downstream temperature is about $3 \mathrm{eV}$.

- The voltage drop across the double layer is about $7 \mathrm{~V}$, and is fairly independent of the external parameters (pressure, power, $\mathrm{SF}_{6}$ concentration).

- The double layers have a spherical shape and resemble a plasma sack attached to the end of the source tube, as described by Andrews and Allen. ${ }^{17}$

\section{THE MODEL}

The experimental situation that we aim to model is shown in Fig. 1(a), while Fig. 1(b) shows the geometry that will be used in the model. We consider that a double layer having a spherical shape of radius $R_{1}=6.5 \mathrm{~cm}$ exists at the interface between the source and the expanding chamber (actually half a sphere). The rf power is deposited in the source plasma, upstream of the double layer, which is modeled as a weakly electronegative cylindrical plasma. The positive ion flux exiting the source is a source term for the downstream plasma. In a similar manner, the upstream plasma is fed with negative ions generated in the downstream plasma.

\section{A. The upstream plasma model}

Experiments have shown that the source plasma is only weakly electronegative. ${ }^{16}$ We therefore use the model developed previously by Kimura et al. ${ }^{18}$ to obtain the two following particle balance equations:

$$
\begin{aligned}
& n_{e} \nu_{i} V-n_{+} n_{-} K_{r} V-n_{e} u_{\mathrm{B}}\left(2 \pi R_{1} L_{1} h_{R}+3 \pi R_{1}^{2} h_{l}\right)=0, \\
& n_{e} \nu_{a} V-n_{+} n_{-} K_{r} V+2 \pi R_{1}^{2} \Gamma_{\mathrm{DL},-}=0,
\end{aligned}
$$

where $\nu_{i}$ and $\nu_{a}$ are functions of the electron temperature $T_{e}$, and are, respectively, the ionization frequency and the attachment frequency, $K_{r}$ is the recombination coefficient, $V$ is the upstream plasma volume, and $\Gamma_{\mathrm{DL},-}$ is the negative ion flux density crossing the double layer from downstream to upstream. Here, and in the downstream region, we do not include detachment, which is usually small compared to recombination with $\mathrm{SF}_{6}$ as the attaching gas. The values of $h_{l}$ and $h_{R}$ (edge-to-central density ratios) have been obtained in Ref. 18 for a relatively low electronegativity cylindrical discharge. Although not completely applicable to our situation, we shall use those values in our calculations. Using charge neutrality, $n_{+}=n_{e}+n_{-}$and introducing the electronegativity $\alpha=n_{-} / n_{e}$, the above equations have four unknowns: $\alpha, n_{e}$, $\Gamma_{\mathrm{DL},-}$, and $T_{e}$. The electron density is determined by the power delivered to the rf antenna and $\Gamma_{\mathrm{DL},-}$ is determined by the downstream plasma equilibrium. These two quantities are input parameters of the upstream model. The first is given by our experimental measurements, and the second is calculated by the downstream model. Then, Eqs. (1) and (2) are solved to obtain $T_{e}$ and $\alpha$.

\section{B. The downstream plasma model}

This model describes the expanding region downstream of the double layer. From experiments, it seems clear that the expanding character leads to strong gradients of the quantities. We therefore include spatial variations in the model by considering that the expanding chamber has a spherical geometry (rather than the actual cylindrical geometry of our experiment) and is terminated at $R_{2} \approx 4.5 R_{1}$. The factor of 4.5 makes the theoretical and experimental area ratios, $A\left(r=R_{2}\right) / A\left(r=R_{1}\right)$ similar. The momentum and particle conservation equations are solved for three coupled fluids in spherical [one-dimensional (1-D)] geometry. We use a fluid model since the mean free path for change exchange in the dominant argon gas is approximately $3 \mathrm{~cm}$ at $P=1 \mathrm{mTorr}$, which is significantly smaller than the chamber dimensions. The three fluids are electrons, positive ions, and negative ions. The electrons are Boltzmann and we consider quasineutrality, $n_{e}+n_{-}=n_{+}$. The electron and ion temperatures are parameters, estimated from experimental results. We define the following quantities:

$$
\begin{aligned}
& \Gamma_{ \pm} \equiv n_{ \pm} u_{ \pm}, \\
& D_{ \pm} \equiv k T_{ \pm} / m_{ \pm} \nu_{ \pm},
\end{aligned}
$$




$$
\gamma_{ \pm} \equiv T_{e} / T_{ \pm}
$$

where $n_{ \pm}$and $u_{ \pm}$are the ion densities and ion fluid velocities, respectively, $T_{e}$ is the electron temperature, $T_{ \pm}$are the ion temperatures, $m_{ \pm}$are the ion masses, and $\nu_{ \pm}$are the ionneutral collision frequencies.

The ion conservation equations in steady state (and in spherical geometry) are

$$
\begin{aligned}
& r^{-2}\left(r^{2} \Gamma_{ \pm}\right)^{\prime}=Q_{ \pm}, \\
& Q_{+}=\nu_{i} n_{e}-K_{r} n_{+} n_{-}, \\
& Q_{-}=\nu_{a} n_{e}-K_{r} n_{+} n_{-},
\end{aligned}
$$

while the momentum conservation equations are

$$
\begin{aligned}
& \Gamma_{ \pm}=D_{ \pm}\left( \pm \frac{q E}{k T_{ \pm}} n_{ \pm}-n_{ \pm}^{\prime}\right), \\
& k T_{e} n_{e}^{\prime}=-e n_{e} E .
\end{aligned}
$$

The electric field may be eliminated and the above equations can be rearranged using charge neutrality and our definitions, to obtain

$$
\left(n_{+}-n_{-}\right) \Gamma_{ \pm}=-D_{ \pm}\left[\left(n_{+}-n_{-}\right) n_{ \pm}^{\prime} \pm \gamma_{ \pm} n_{ \pm}\left(n_{+}-n_{-}\right)^{\prime}\right],
$$

which is equivalent to

$$
\begin{aligned}
\left(\begin{array}{c}
n_{+}^{\prime} \\
n_{-}^{\prime}
\end{array}\right) & \\
= & \frac{\left(\begin{array}{cc}
-D_{-}\left(n_{+}-n_{-}\right)-D_{-} \gamma_{-} n_{-} & -D_{+} \gamma_{+} n_{+} \\
-D_{-} \gamma_{-} n_{-} & -D_{+} \gamma_{+} n_{+}-D_{+}\left(n_{+}-n_{-}\right)
\end{array}\right)}{D_{-} D_{+}\left[\left(1+\gamma_{+}\right) n_{+}-\left(1-\gamma_{-}\right) n_{-}\right]} \\
& \times\left(\begin{array}{l}
\Gamma_{+} \\
\Gamma_{-}
\end{array}\right) .
\end{aligned}
$$

These two equations, along with the ion conservation equations [Eqs. (6)-(8)], lead to the following system of four first order ordinary differential equations:

$$
\begin{aligned}
& n_{+}^{\prime}=\frac{D_{-}\left[\gamma_{-} n_{-}-\left(n_{+}-n_{-}\right)\right] \Gamma_{+}+D_{+} \gamma_{+} n_{+} \Gamma_{-}}{D_{-} D_{+}\left[\left(1-\gamma_{-}\right) n_{-}-\left(1+\gamma_{+}\right) n_{+}\right]}, \\
& n_{-}^{\prime}=\frac{D_{-} \gamma_{-} n_{-} \Gamma_{+}+D_{+}\left[\gamma_{+} n_{+}+\left(n_{+}-n_{-}\right)\right] \Gamma_{-}}{D_{-} D_{+}\left[\left(1-\gamma_{-}\right) n_{-}-\left(1+\gamma_{+}\right) n_{+}\right]}, \\
& \Gamma_{+}^{\prime}=\nu_{i}\left(n_{+}-n_{-}\right)-K_{r} n_{+} n_{-}-\frac{2 \Gamma_{+}}{r}, \\
& \Gamma_{-}^{\prime}=\nu_{a}\left(n_{+}-n_{-}\right)-K_{r} n_{+} n_{-}-\frac{2 \Gamma_{-}}{r} .
\end{aligned}
$$

These equations are normalized and solved numerically for given electron and ion temperatures. Together with the basic equations, one needs to define the appropriate boundary conditions at $r=R_{1}$ and $r=R_{2}$. We use the following set of conditions:

$$
\Gamma_{+}\left(r=R_{1}\right)=\Gamma_{0}=h_{l} n_{e 0} u_{\mathrm{B}},
$$

$$
\Gamma_{-}\left(r=R_{2}\right)=0
$$

$$
u_{-}\left(r=R_{1}\right)=u_{\mathrm{B}} / \sqrt{\gamma_{-}} \text {, }
$$

$$
u_{+}\left(r=R_{2}\right)=u_{\mathrm{B}} / \sqrt{\gamma_{-}},
$$

where $n_{e 0}$ is the electron density in the center of the source and $u_{\mathrm{B}}=\left(k T_{e} / m_{+}\right)^{1 / 2}$ is the Bohm velocity. Condition (17) implies that the positive ions entering the expanding region through the double-layer flow from the source region into the expanding region, with the flux $\Gamma_{0}$, obtained from (1), without radial variations. This flux is determined by the measured (input) upstream electron density and from the appropriate $h_{l}$ factor from the upstream solution. The assumption is made that the negative ions flowing into the source region do not create sufficient local negative ion density to modify the Bohm velocity. The positive ions (created in the downstream region and flowing from the double layer) reach the bottom of the expanding chamber (at $r=R_{2}$ ) and exit with a Bohm velocity that may be reduced due to the electronegativity downstream [condition (20)]. Here we have assumed that the Bohm velocity is reduced [as suggested by experimental evidence in Fig. 3(b), later] but we will examine this assumption again in Sec. IV. The other assumptions are relatively straightforward. The flux of negative ions at $r=R_{2}$ must be zero [condition (18)] due to the sheath at the expanding chamber walls, and a reduced Bohm velocity for negative ions entering the double layer [condition (19)] is reasonable because of the high downstream electronegativity there.

\section{The double layer: A permeable boundary}

The two models are coupled via the double layer that acts as a permeable internal sheath. Some of the positive ions generated upstream flow downstream through the double layer while some of the negative ions generated downstream flow through the double layer toward the source. Using the electron density in the center of the source $n_{e 0}$, and the value of $h_{l}$ determined from the model, the upstream model electron density just before the double layer is $h_{l} n_{e 0}$. Just downstream from the double layer, the electron density is $n_{e}\left(r=R_{1}\right)$, as calculated by the system of Eqs. (13)-(16) together with the boundary conditions (17)-(20). For selfconsistency, if the electron energy distribution function is Maxwellian, the double-layer voltage would be related to the electron densities upstream and downstream of the double layer by the Boltzmann relation,

$$
V_{\mathrm{DL}}=T_{e} \ln \left(\frac{h_{l} n_{e 0}}{n_{e}\left(r=R_{1}\right)}\right) \text {, }
$$

with $T_{e}$ a uniform temperature across the double layer. However, experiments have shown that the electron temperature is different in the source and in the downstream region; we will come back to this in Sec. IV. 
TABLE I. Elastic and inelastic collision frequencies.

\begin{tabular}{lcc}
\hline \hline Frequency & Expression & Value in s ${ }^{-1}$ at $1.5 \mathrm{mTorr}, T_{e}=3 \mathrm{eV}$ \\
\hline$\nu_{i}$ & $n_{g} K_{i 0} T_{e}^{0.59} \exp \left(-17.44 / T_{e}\right)$ & $6.4 \times 10^{3}$ \\
$\nu_{a}$ & $\nu_{a}=n_{g} K_{\mathrm{a} 0} /\left(T_{e}^{1.2}\right)$ & $1.6 \times 10^{4}$ \\
$\nu_{-}$ & $n_{g} \sigma_{\mathrm{iN}} \bar{v}_{-}$ & $1.4 \times 10^{4}$ \\
$\nu_{+}$ & $n_{g} \sigma_{\mathrm{iN}} \bar{v}_{+}$ & $3.75 \times 10^{4}$ \\
$\nu_{r}$ & $n_{+} K_{r}$ & Typically $10^{3}$ \\
\hline \hline
\end{tabular}

\section{COMPARISONS BETWEEN MODEL AND EXPERIMENTS}

We first ran the model for similar conditions as in the experiments published in Plihon et al. ${ }^{16}$ The total pressure when the plasma is on is $p=1.5 \mathrm{mTorr}$ ( $1 \mathrm{mTorr}$ with plasma off), the gas composition is $92 \%$ argon, $8 \% \mathrm{SF}_{6}$, and the rf power applied to the antenna is $600 \mathrm{~W}$. We consider argon positive ions, with mass $m_{+}=40 \mathrm{amu}$, and a mixture of $\mathrm{F}^{-}$and $\mathrm{SF}_{5}^{-}$negative ions (50\% each), with an effective mass of $m_{-}=73 \mathrm{amu}$. The positive ion temperature is fixed at $T_{+}=0.1 \mathrm{eV}$, while the negative ion temperature is taken to be higher at $T_{-}=0.3 \mathrm{eV}$, as typically proposed for low-pressure electronegative discharges. ${ }^{8,19}$ The expressions for ionization, attachment, recombination, and ion-neutral collision frequencies are summarized in Table I. We chose argon cross sections given in Ref. 1 for ionization, with $K_{i 0}=2.34$ $\times 10^{-14}$. For the attachment rate, we chose $K_{a 0}=2 K_{a 0, \mathrm{SF}_{6}} \beta$, where $\beta$ is the $\mathrm{SF}_{6} /\left(\mathrm{Ar}+\mathrm{SF}_{6}\right)$ ratio (for the $1.5 \mathrm{mTorr}$ case discussed here, $\beta=0.08$ ) and $K_{a 0, \mathrm{SF}_{6}}=7.76 \times 10^{-15}$ is the value in $\mathrm{SF}_{6}$. This attachment rate expression was chosen as the best fit for the 1.5 mTorr case, and was used regardless of the pressure and the power. Note that this value is two times larger than one would expect for $\mathrm{SF}_{6}$ nondissociated. However, this choice does not seem unreasonable since the attachment on fragments might be higher than on $\mathrm{SF}_{6}$. Finally, $K_{r}=3 \times 10^{-14} \mathrm{~m}^{3} \mathrm{~s}^{-1}$. For this pressure, values of $h_{l}=h_{R}=0.5$ were chosen to calculate the upstream equilibrium. From the results given in Ref. 18 these quantities vary from 0.6 to 0.45 when the pressure increases from 0.5 to 6 mTorr. The source temperature is fairly insensitive to these values, but $h_{l}$ at the double layer must be known fairly accurately.

As described in Sec. II, the two input parameters for the equilibrium model are the measured electron density in the center of the source (upstream) region, and the measured electron temperature in the expanding (downstream) region. For the case described above, these values are $n_{e 0}=10^{17} \mathrm{~m}^{-3}$ and $T_{e}=3 \mathrm{eV}$, respectively, as obtained from probe measurements. Note that the upstream electron density could be calculated from the input power, but the upstream equilibrium was more directly calculated using the measured central density. The upstream temperature follows in a straightforward manner from the equilibrium equations (1) and (2), with little effect from the downstream equilibrium. From these two equations the electron temperature in the source is calculated to be $T_{e}=4.5 \mathrm{eV}$, in good agreement with the experimental value. Since the electron temperature is, as measured discontinuous across the double layer, this

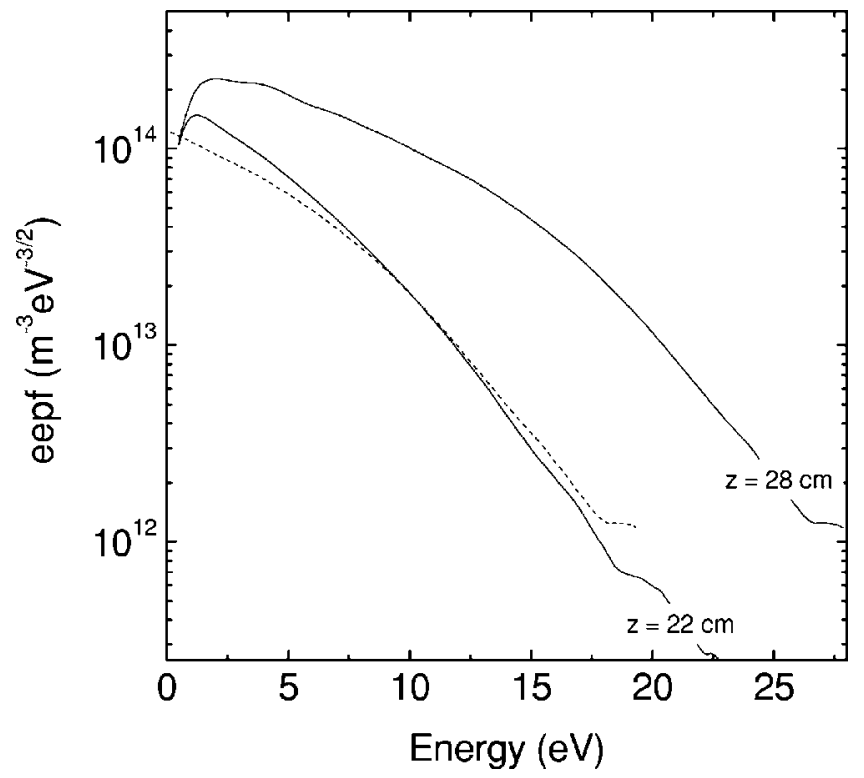

FIG. 2. Experimental electron energy probability function (eepf) at $z=22 \mathrm{~cm}$ (downstream of the double layer) and at $z=28 \mathrm{~cm}$ (upstream of the double layer). The dashed line is the upstream eepf shifted by $8.5 \mathrm{~V}$.

implies that the upstream electron energy distribution function (eedf) is non-Maxwellian. We can investigate this from the observed electron energy probability function (eepf) in the source and downstream regions as shown in Fig. 2. These eepf's were derived in the usual way from our Langmuir probe I-V curves. A Boltzmann shift of the upstream distribution passing through the double layer cuts off the lower energy (but higher effective $T_{e}$ ), with an $8.5 \mathrm{~V}$ shift, bringing the upstream distribution into coincidence with the downstream distribution (dashed curve). This is reasonably consistent with a measured double-layer voltage of $7.5 \mathrm{~V}$, plus an additional shift on the source side due to the existence of a presheath.

Downstream, using Eqs. (13)-(16) along with the boundary conditions (17)-(20), the theoretical values of the electron density, and the electronegativity, are compared to the experimental values. Figure 3(a) shows the electron density, and Fig. 3(b) shows the electronegativity $\alpha$ as a function of the axial position in the expanding region. The circles give the experimental values while the lines are the theoretical results. The coordinate $z=0$ corresponds to $r=R_{2}=4.5 R_{1}$ in the calculation, and, similarly, $z \approx 22.5 \mathrm{~cm}$ corresponds to $r=R_{1}=6.5 \mathrm{~cm}$. The absolute values of both the electron density and electronegativity are in good agreement, although the gradient in the electron density is steeper for the experiments. Both the electron density and the electronegativity decay from the downstream edge of the double-layer position to the bottom of the chamber. This is well captured by the model and is mainly due to the expansion. Although it is possible to give a profile of the source electrons, as given in Refs. 18, we have not done this, but have only used the $h_{l}$ factor. The value of $h_{l} n_{e 0}$ upstream and the value immediately downstream are reasonably consistent with the experiment. From the experimental technique of measuring $\alpha$, the values given in Fig. 3(b) are upper bounds and become in- 


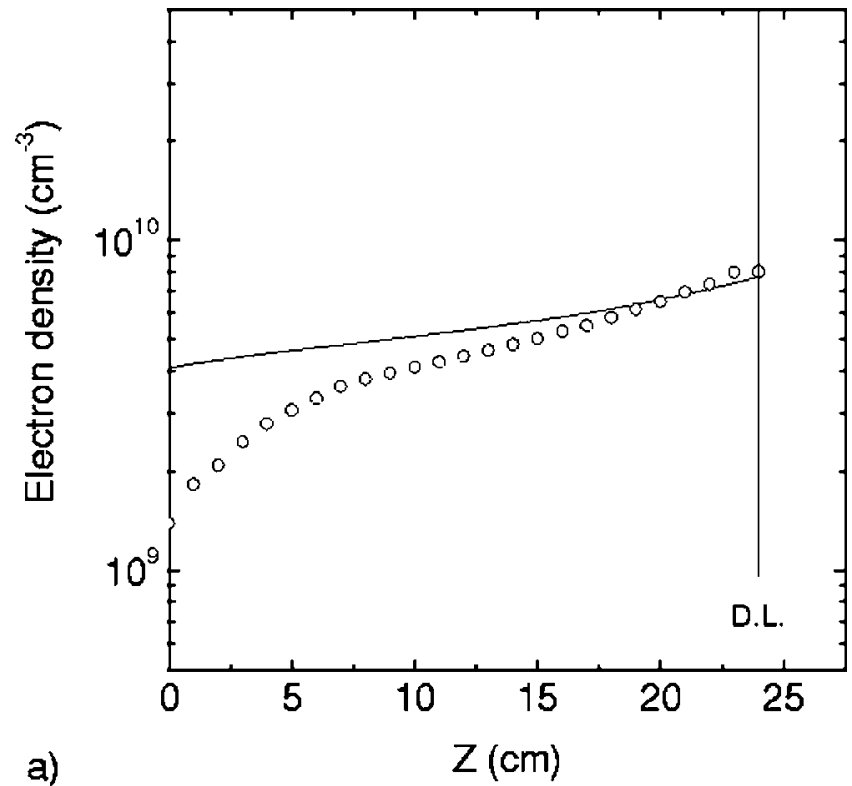

a)

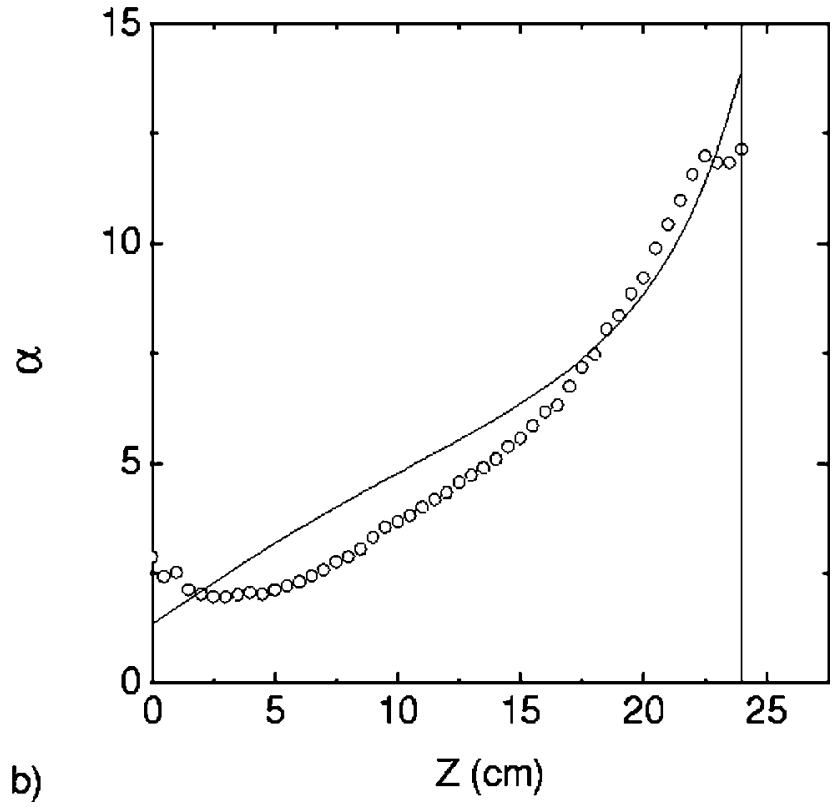

FIG. 3. Electron density (a) and electronegativity (b) as a function of the altitude in the expanding chamber. The pressure was $1.5 \mathrm{mTorr}$, the $\mathrm{SF}_{6} / \mathrm{Ar}$ ratio was $8 \%$, and the rf power was $600 \mathrm{~W}$. The symbols stand for the experiments while the lines stand for the model.

creasingly uncertain below $\alpha \approx 2 .{ }^{19}$ The model indicates an electronegativity in the center of the source region of approximately $\alpha \approx 2$.

We now compare the model and experimental scalings when changing the three input parameters, rf power, pressure, and $\mathrm{SF}_{6}$ concentration. Figure 4 shows the downstream electron density (taken at $z=15 \mathrm{~cm}$ ) as a function of the electron density in the middle of the source. This curve was obtained by increasing the rf power for similar pressure and gas composition conditions as above. The densities increase with increasing $\mathrm{rf}$ power (as expected), accompanied by weak changes in electronegativity and electron temperature. The model (solid line) is in excellent quantitative agreement with experiments. When increasing the $\mathrm{SF}_{6}$ concentration,

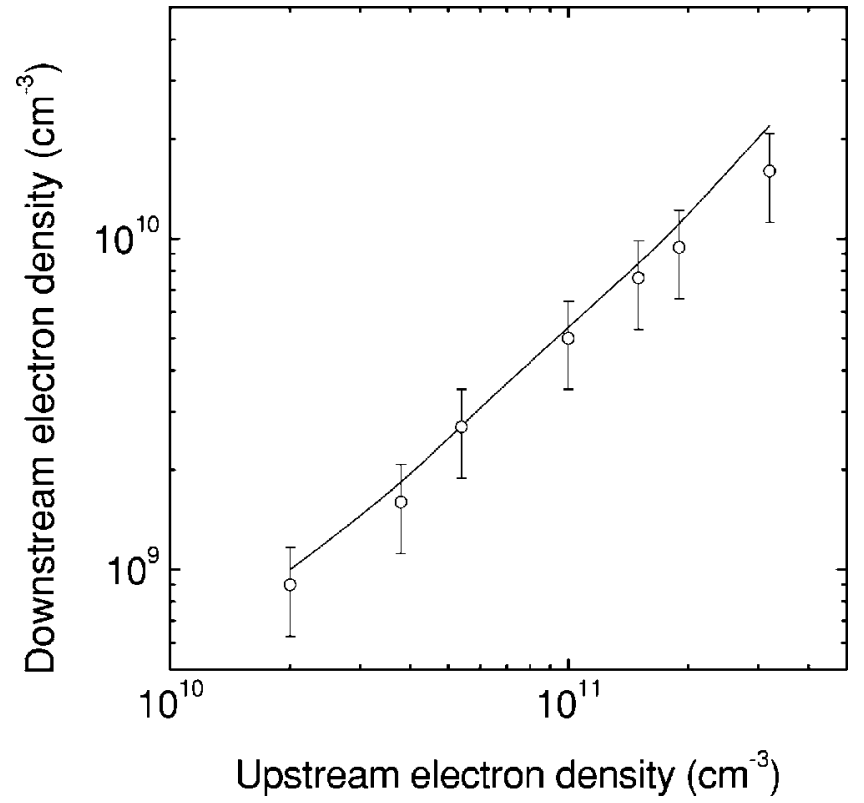

FIG. 4. Downstream electron density (at $z=15 \mathrm{~cm}$ ) versus upstream electron density (in the middle of the source) for the same conditions as previously. The increase in upstream density was obtain by increasing the rf power.

we rapidly entered an unstable regime with the experimental results given in Ref. 7. This regime cannot be described by the current model and therefore we do not explore a wide range of $\mathrm{SF}_{6}$ concentration in this comparison of steady state results.

The changes in pressure led to significant changes of the equilibria, as discussed in the following. Figure 5(a) shows the electron density, Fig. 5(b) shows the electron temperature, and Fig. 5(c) shows the electronegativity $\alpha$, all as a function of pressure (when the plasma is on). Again, these measurements were taken at $z=15 \mathrm{~cm}$. Note that in order to keep the conditions for a stationary double layer, the $\mathrm{SF}_{6}$ concentration had to be decreased from $15 \%$ at 0.5 mTorr to $5.5 \%$ at 6.1 mTorr (we accounted for this change in calculating the attachment rate, via the $\beta$ factor). The electron temperature is an input of the downstream model, which allows one to calculate the electron density and the electronegativity. The calculated electron density is in very good agreement with measurements. The plasma becomes more electronegative as the pressure increases, which leads to the decay in the electron density [see Fig. 5(a)] as well as the increase in $\alpha$, as seen in Fig. 5(c). Note that the agreement between theory and experiment is not as good when comparing electronegativity $\alpha$, although the trends are respected. This discrepancy might be due to the change in attachment rate due to dissociation (the model only account for the $\mathrm{SF}_{6}$ concentration before the plasma is struck).

The upstream quantities are shown in Fig. 6 for the same conditions as in Fig. 5. The upstream electron density (in the middle of the source) slightly decays with pressure, due to an increase in the attachment rate. This is an input parameter of the model. The electron temperature decreases with pressure, as expected, and we note very good agreement between theory and experiment. The electronegativity increases with pressure, which is also reasonable as the attaching rate in- 

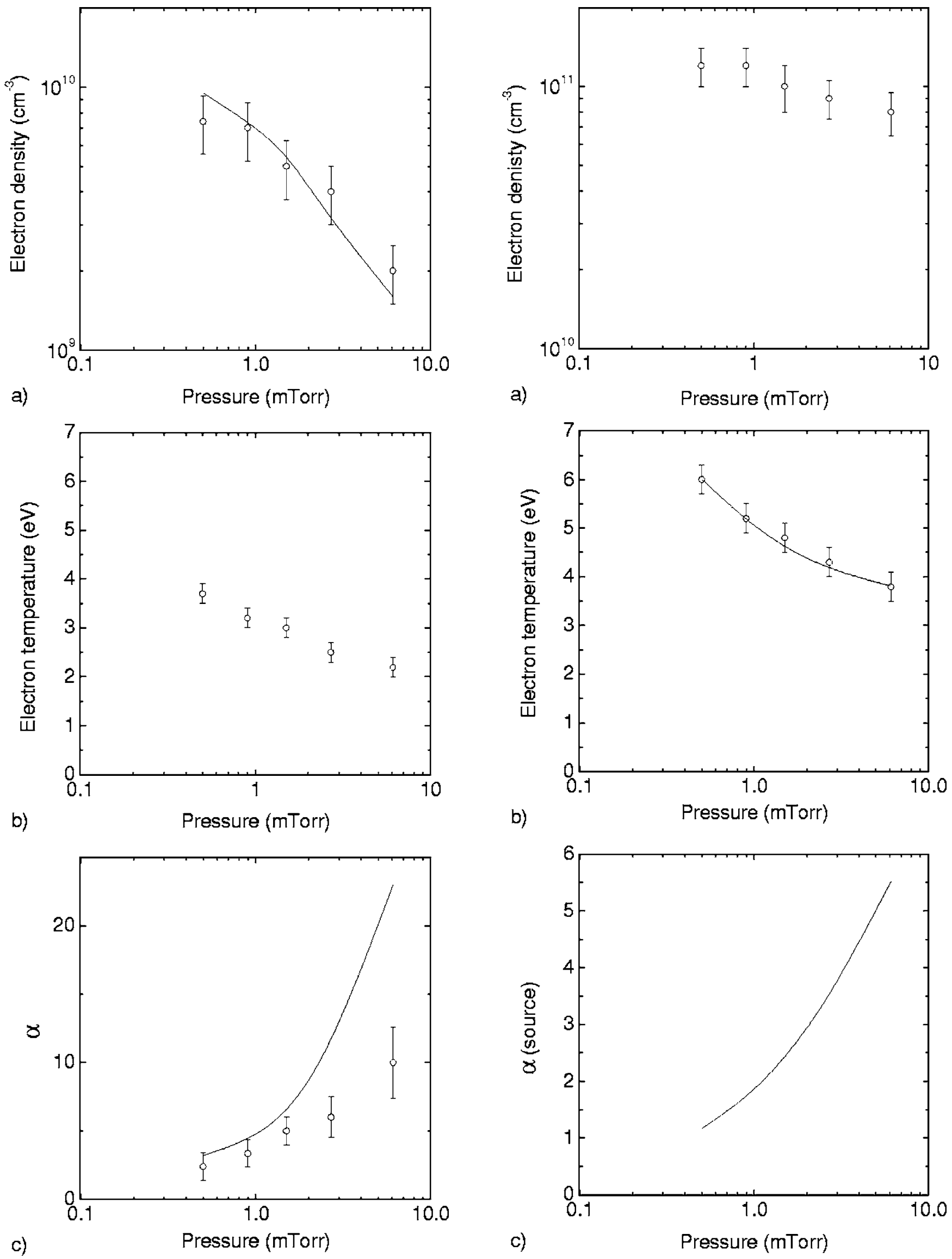

FIG. 5. Electron density (a), electron temperature (b), and electronegativity (c) in the center of the expanding chamber as a function of pressure. The symbols stand for the experiments while the lines are for the model. The rf power was $600 \mathrm{~W}$.

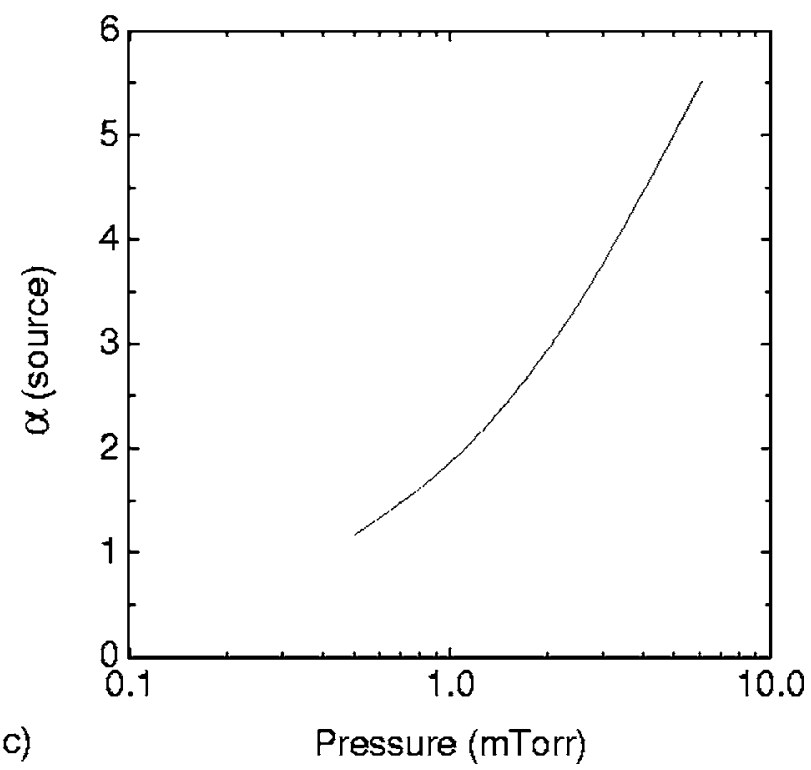

FIG. 6. Electron density (a), electron temperature (b), and electronegativity (c) in the center of the source and as a function of pressure, and for the same conditions as in Fig. 5. The symbols stand for the experiments while the lines are for the model. 


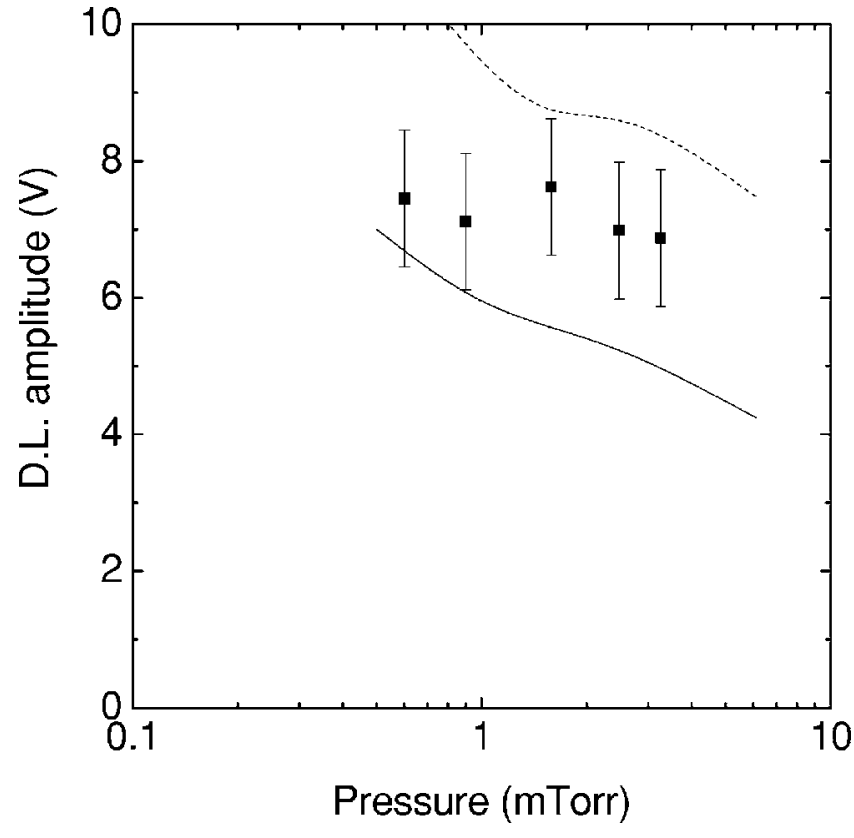

FIG. 7. Double-layer amplitude as a function of pressure for the same conditions as in Fig. 5. The symbols stand for experiments, the dotted lne is the calculated amplitude using the upstream electron temperature, while the solid line is the calculated amplitude using the downstream electron temperature.

creases. Note that we could not make electronegativity measurements in the middle of the source.

To conclude this section, we compare in Fig. 7 the calculated and measured double-layer amplitude as a function of pressure. The solid line gives the calculated amplitude when using the downstream temperature in Eq. (21) while the dotted line gives the calculated amplitude when using the upstream temperature. The experimental values lie between these two curves, at about 7-8 V, showing little amplitude variations as the pressure changes. As might be expected from a Boltzmann condition, the experimental values correspond to temperatures between those upstream and downstream. The detailed relationships depend on the changing distribution functions with pressure and merit a more detailed investigation.

\section{CONCLUSIONS AND FURTHER DISCUSSION}

We have developed a model to describe the equilibrium of a low-pressure electronegative discharge having an internal double layer separating the source plasma from the expanding plasma. The model agrees reasonably well with experiments and reproduces all the general trends. In particular, the model indicates that the gradients of plasma parameters downstream of the double layer are mainly due to the geometrical expansion. The model tends to overestimate the values of $\alpha$ downstream, particularly giving values that increase too rapidly with increasing pressure. The simplest global estimate of the downstream scaling of $\alpha$ with pressure is given from an equilibrium relation, at low density,

$$
\int n_{e} \nu_{a} d \tau=2 \pi R_{1}^{2} \Gamma_{\mathrm{DL},-},
$$

where recombination is neglected. This relation gives a scaling, weakly dependent on $T_{e}$, of $\alpha$ proportional to $n_{g}$, with recombination only weakening the proportionality. This proportionality is essentially observed experimentally, but is somewhat stronger in the theoretical model, as seen in Fig. 5(c). Another difference between the model and the experimental result is the more rapid experimental falloff of the electron density near $r=R_{2}(z=0)$ compared to the model, as seen in Fig. 3(a). This is accompanied by a surprising observed increase of the experimental $\alpha$ at the plasma edge. This led to a reduced positive ion Bohm velocity at $r=R_{2}$ [boundary condition (19)]. However, as previously noted, the smaller measured values of $\alpha$ are unreliable and may be considerably smaller at low $\alpha$ values. From the previous theory developed in Ref. 18, we would expect to have an electropositive edge, and therefore we would replace the boundary condition (20) with the condition $u_{+}=u_{\mathrm{B}}$, i.e., the ions would exit with the full Bohm velocity. We have compared the theoretical electron density with this boundary condition to the results obtained in Fig. 3(a) and find a more rapid falloff of $n_{e}$ near $r=R_{2}$, as seen experimentally. The result is shown in Fig. 8. Although this new boundary condition changes the gradient of $n_{e}$ near the lower boundary (at $r=R_{2}$ ), it does not profoundly modify the overall values shown in Figs. 2-7 and consequently the major conclusions of this work.

We can also use the model to gain insight into the nature of the equilibria by examining the importance of the coupling terms. Figure 9 shows the calculated ion fluxes in the downstream plasma for the 1.5 mTorr case. The negative ion flux is about $70 \%$ of the positive ion flux across the double layer at this pressure. As the pressure changes from 0.5 to 6 mTorr this percentage changes from about 30\% at low pressure to nearly $100 \%$ at high pressure. However, the negative ion flux coming from downstream does not necessarily dominate the negative ion balance equation [Eq. (2)] upstream. Indeed, the ratio

$$
x=\frac{2 \pi R_{1}^{2} \Gamma_{\mathrm{DL},-}}{\int n_{e} \nu_{a} d \tau}
$$

(where the denominator is the integral over the volume of the attachment rate downstream) falls from about one at 0.5 mTorr, where the downstream negative ions contribute to the equilibrium, to 0.08 at 6 mTorr, where they barely play a role.

Similarly, one can examine the importance of positive ions coming from the source for the downstream equilibrium. For this, we look at the following ratio:

$$
y=\frac{2 \pi R_{1}^{2} \Gamma_{0}}{\int n_{e} \nu_{i} d \tau},
$$

where the denominator is the integral over the volume of the ionization rate downstream. As the pressure changes from 0.5 to 6 mTorr, $y$ changes from about 1 to nearly 30. Therefore, the positive ions generated upstream rapidly dominate 


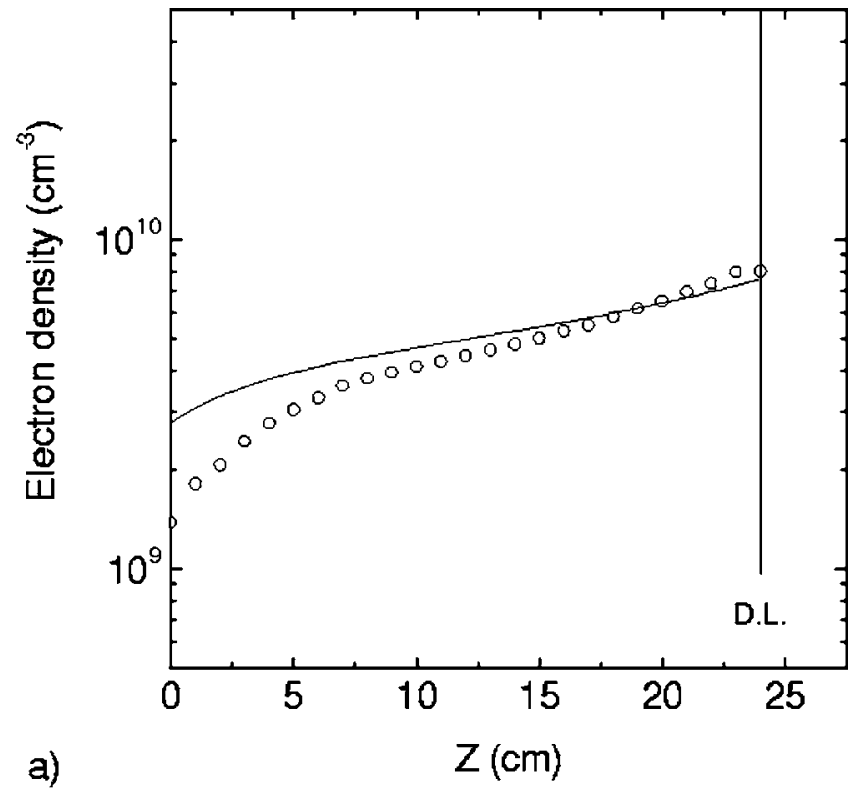

a)

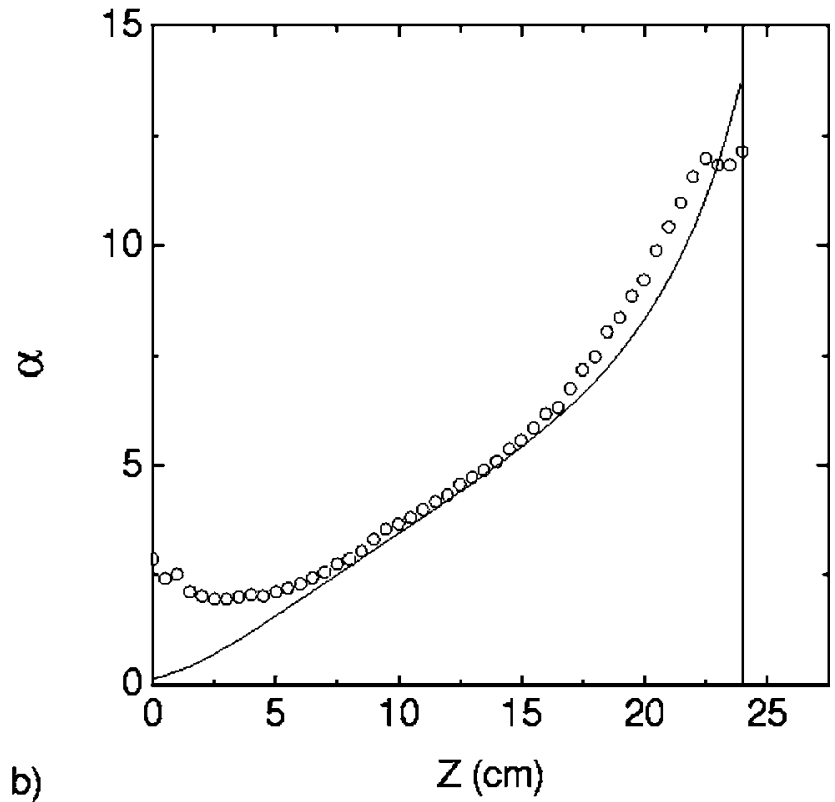

FIG. 8. The same as Fig. 3 calculated with the new boundary condition $u_{+}\left(r=R_{2}\right)=u_{\mathrm{B}}$.

as the pressure increases, mainly because the downstream electron temperature decreases such that local ionization becomes negligible. Thus, at very low pressure (typically $0.5 \mathrm{mTorr})$, the two plasma equilibria are strongly coupled by the double layer. The negative ions created downstream contribute to the upstream equilibrium as the upstream positive ions contribute to the downstream equilibrium (without being dominant). At higher pressure, the situation becomes strongly asymmetric. The upstream plasma is not modified by the downstream plasma (i.e., by the negative ions coming from downstream), whereas the downstream plasma equilibrium is governed by the positive ions coming from the source (i.e., local ionization is negligible).

As mentioned in Sec. II the model comparison is a selfconsistency calculation and not a direct calculation of the double layer within a complete theory. To understand the

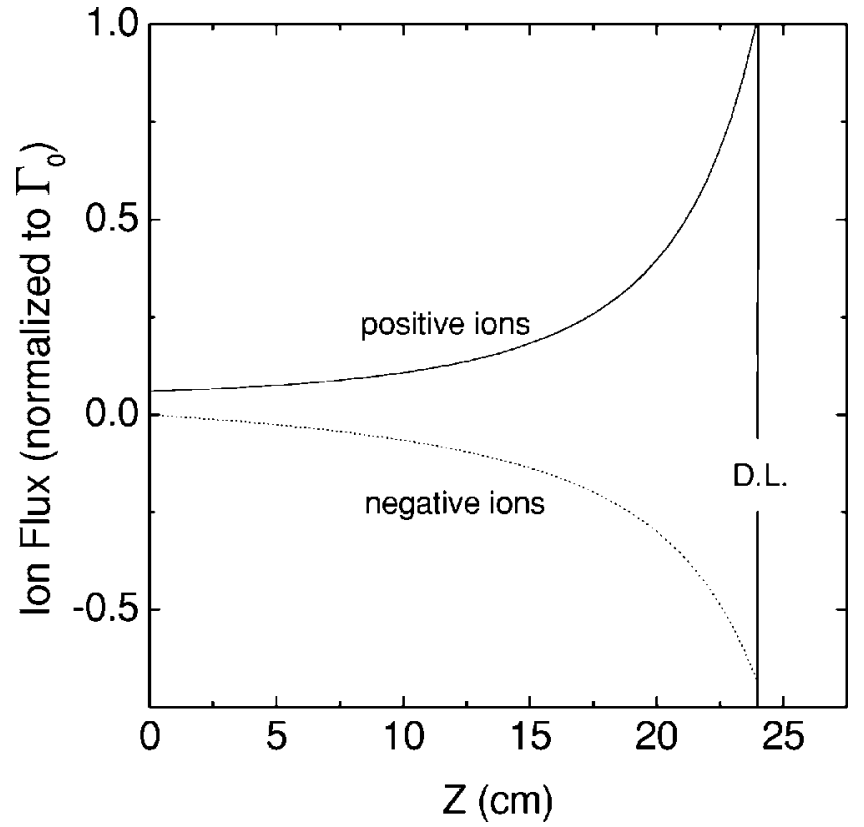

FIG. 9. Ion fluxes in the expanding chamber as calculated by the model.

detailed physics of the double-layer region, one needs to measure the electron energy distribution function and to incorporate the electron dynamics, as well as Poisson's equation to describe the space charge and potential variations.

\section{ACKNOWLEDGMENTS}

The authors gratefully acknowledge fruitful discussions with Mike Lieberman and Raoul Franklin.

This work has been supported by the European Space Agency, under Ariadna Study Contract No. ACT-04-3101. One of the authors (A.J.L.) acknowledges the hospitality of the LPTP, where the collaboration was begun.

\footnotetext{
${ }^{1}$ M. Lieberman and A. Lichtenberg, Principles of Radiofrequency Discharges and Material Processing (Wiley, New York, 2005), 2nd ed..

${ }^{2}$ M. Lieberman, A. J. Lichtenberg, and A. M. Marakhtanov, Appl. Phys. Lett. 75, 3617 (1999).

${ }^{3}$ P. Chabert, A. J. Lichtenberg, M. Lieberman, and A. M. Marakhtanov, Plasma Sources Sci. Technol. 10, 478 (2001).

${ }^{4}$ P. Chabert, H. Abada, J.-P. Booth, and M. Lieberman, J. Appl. Phys. 94, 76 (2003).

${ }^{5}$ P. Chabert, A. Lichtenberg, M. Lieberman, and A. Marakhtanov, J. Appl. Phys. 94, 831 (2003).

${ }^{6}$ A. Marakhtanov, M. Tuszewski, M. L. A. Lichtenberg, and P. Chabert, J. Vac. Sci. Technol. A 21, 1849 (2003).

${ }^{7}$ N. Plihon, C. Corr, P. Chabert, and J.-L. Raimbault, J. Appl. Phys. 98, 023306 (2005)

${ }^{8}$ M. Tuszewski, J. Appl. Phys. 79, 8967 (1996).

${ }^{9}$ M. Tuszewski, R. R. White, and G. A. Wurden, Plasma Sources Sci. Technol. 12, 396 (2003).

${ }^{10}$ M. Tuszewski and R. R. White, J. Appl. Phys. 94, 2858 (2003).

${ }^{11}$ M. Tuszewski and S. P. Gary, Phys. Plasmas 10, 539 (2003).

${ }^{12}$ I. Kouznetsov, A. Lichtenberg, and M. Lieberman, J. Appl. Phys. 86, 4142 (1999).

${ }^{13}$ T. Sheridan, P. Chabert, and R. Boswell, Plasma Sources Sci. Technol. 8, 457 (1999).

${ }^{14}$ R. Merlino and J. Loomis, Phys. Fluids B 2, 2865 (1990).
} 
${ }^{15}$ T. Sheridan, N. Braithwaite, and R. Boswell, Phys. Plasmas 6, 4375 (1999).

${ }^{16}$ N. Plihon, C. Corr, and P. Chabert, Appl. Phys. Lett. 86, 091501 (2005).

${ }^{17}$ J. Andrews and J. Allen, Proc. R. Soc. London, Ser. A 320, 459 (1971).
${ }^{18}$ T. Kimura, A. Lichtenberg, and M. Lieberman, Plasma Sources Sci. Technol. 10, 430 (2001).

${ }^{19}$ P. Chabert, T. Sheridan, R. Boswell, and J. Perrin, Plasma Sources Sci. Technol. 8, 561 (1999). 